\title{
First principle calculations with SIC correction of Fe-doped $\mathrm{CuO}$ compound
}

\author{
Fatima Zahra $\mathrm{CHAFI}^{{ }^{*}}{ }^{*}$, Elmehdi SALMANI ${ }^{2}$, Lahoucine BAHMAD ${ }^{2}$, Najem HASSANAIN $^{1}$, Fares \\ BOUBKER $^{l}$, and Ahmed MZERD ${ }^{l}$ \\ ${ }^{1}$ Laboratory of Physics and Materials (LPM) - Mohammed V University, Faculty of Science- BP. 1014- Av. Ibn Batouta \\ Rabat, Morocco \\ ${ }^{2}$ Laboratory of Magnetism and Physics of High Energy (LMPHE-URAC-12) - Mohammed V University, Faculty of Science - BP. 1014- Av \\ Ibn Batouta Rabat, Morocco \\ *Corresponding authore-mail address: chafifatimazahra@gmail.com
}

\begin{abstract}
In this work, the electronic properties of Fe-doped $\mathrm{CuO}\left(\mathrm{Cu}_{1-x} \mathrm{Fe}_{\mathrm{x}} \mathrm{O}\right)$ thin films are studied by using a standard density functional theory (DFT) based on the ab-initio approach under the Korringa-KohnRostoker coherent potential approximation (KKR-CPA). This study is carried out in the framework of the general gradient approximation (GGA) and self-interaction-corrected (SIC). The density-of-states (DOSs) in the energy diagram are illustrated and discussed. The computed electronic properties of our compounds confirm the half-metalicity nature of this material $(\mathrm{CuO})$. In addition, the absorption spectra of the studied compound within the Generalized Gradient Approximation GGA, as proposed by Perdew-BurkeErnzerhof (PBE) and GGA-PBE -SIC approximations are examined. Compared with the pure $\mathrm{CuO}$, the Fermilevels of doped structures are found to move to the higher energy directions. Finally, the effect of Fe-doping method in $\mathrm{CuO}$ can transform the material to half-metallic one, with a high wide impurity band in two cases of approximations local density approximation (LDA) and SIC method.
\end{abstract}

Keywords: Ab-initio calculation; Fe-doped CuO; KKR-CPA; GGA; SIC correction; Half-metalicity. 


\section{Introduction}

The diluted magnetic semiconductors (DMS) are the basis of new materials. They have shown interesting results for new generation of spintronics devices. These materials are obtained by the substitution of a nonmagnetic semiconductor by transition metal (TM) elements such as $\mathrm{Co}, \mathrm{Mn}, \mathrm{Ni}, \mathrm{Cr}$ and $\mathrm{Fe}$ [1]. The DMSs showed a high Curie temperature $T_{c}$ and half-metallic behaviors. As well as other transition metal oxides, the Copper Oxide $(\mathrm{CuO})$ material has anti-ferromagnetic ground state [2]. As a narrow band gap semiconductor, the $\mathrm{CuO}$ has been proved to be a good host material for diluted magnetic semiconductors $[3,4]$.

Recently, extensive studies have been carried out in transition elements-doped $\mathrm{CuO}$ on account of their potential uses in many technological fields such as: spintronics [5-7], memory devices [8], optoelectronics [9], gas sensors [10-13], solar cells [14] and more other applications [15-23].

To study the electronic properties of $\mathrm{CuO}$, many theoretical techniques have been used like B3LYP (Becke three parameters, Lee, Yang and Parr) [24], PWPP-LSDA (Plane Wave Pseudo Potential-Local Spin Density Approximation) [25], CIPSI (Configuration Interaction by Perturbation Selected Iteratively) [26], CASSCF (Complete Active Space Self Consistent Field) [27], LSDA (Local Spin Density Approximation) [28] and PBEGGA (Perdew functional with Generalized Gradient Approximation) [29] methods.

In this paper, we are interesting in the study of electronic properties of $\mathrm{Cu}_{1-\mathrm{x}} \mathrm{Fe}_{\mathrm{x}} \mathrm{O}$ for specific concentration values $(\mathrm{x}=0.05,0.10,0.15)$, using the density function theory (DFT) under the Korringa-Kohn-Rostoker (KKR) method as well as the coherent potential approximation (CPA) with LDA (local density approximation) and SIC (self-interaction-corrected) approximations. This article is organized as follows: in next section we discuss the method and computational details. Section 3 illustrates a sketch of geometry of the studied compound. In section 4, we give results and discussion. Section 5 is devoted to a conclusion of this work.

\section{Computational details}

We use the Korringa-Kohn-Rostoker (KKR) method combined with the coherent potential approximation (CPA) within the local spin density approximation (LSDA) to study the electronic structure. This has the advantage of taking into account the randomness of the impurity elements. To parameterize the exchange energy we used the Generalized Gradient approximation (GGA) [30]. For more realistic description of the disordered local moments of the materials under investigation, the self-interaction corrected "SIC" approach is applied. This method is developed by Toyoda [31] within the KKR-CPA-SIC-GGA package as implemented into MACHIKANEYAMA2002 [32]. Due to hybridization of the orbital with the valance band and the energy gain upon localization of the orbital, the SIC approximation is found to be governed by the energy difference between the energy gain [33]. Compared to the GGA approximation, the SIC-GGA leads to an enhancement of the photoemission spectra. Free calculation of the SIC-GGA is similar to the GGA + U approximation [34]. The correction $\mathrm{U}$ is taken into account to fit the photoemission spectra in the dilute magnetic semiconductors (DMS). One of the most efficient methods is the CPA approximation which is considered to be one of the most used methods to study the band structure calculations. This method was developed by Akai and Dederichs [35], to investigate the transition metal alloys in As-based DMS [36]. 


\section{Crystal Structure}

Copper oxide $(\mathrm{CuO})$ is commonly known as a Tenorite and p-type semiconductor with a band gap of $1.2 \mathrm{eV}$ [37]. The crystal structure of $\mathrm{CuO}$ is monoclinic (see figure1) with $\mathrm{C} 2 / \mathrm{c}$ symmetry, the dimension of unit cell is a= $4.69 \AA, b=3.43 \AA, c=5.15 \AA, \alpha=\gamma=90^{\circ}$ and $\beta=99.52^{\circ}$. The copper ions occupy positions $\pm(1 / 4,1 / 4,0 ; 3 / 4,1 / 4$, $1 / 2$ ) along the lattice vectors, and the oxygen ions take positions $\pm(0, u, 1 / 4 ; 1 / 2, u+1 / 2,1 / 4)$, where $u=-0.584$ is a parameter describing the relative positions of oxygen ions along this lattice.

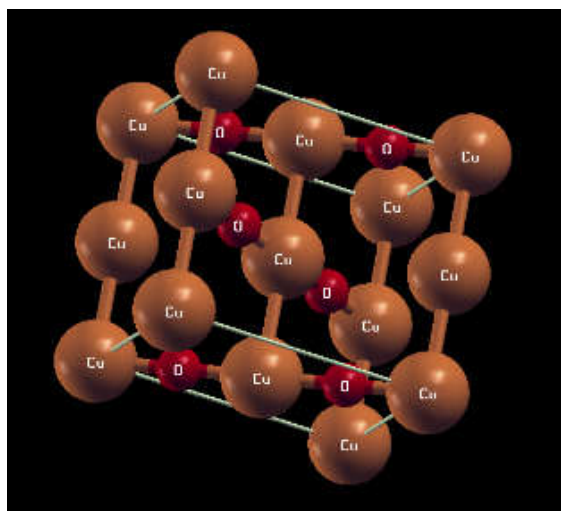

Figure 1. A sketch of the geometry of the monoclinic structure of pure $\mathrm{CuO}$. The red circles represent Oxygen atoms and the brown ones represent the Copper atoms.

\section{Results and discussion}

\subsection{Electronic structure}

We generate the coherent potential approximation (CPA) from the atomic configuration of [Ar], for Cu $3 d^{10} 4 s^{1}$. The valence electrons used are $3 \mathrm{~d}$ and $4 \mathrm{~s}$. In order to generate the coherent potential (CPA) approximation for O, we use $[\mathrm{He}] 2 \mathrm{~s}^{2} 2 \mathrm{p}^{4}$. The valence electrons used are $2 \mathrm{~s}$ and $2 \mathrm{p}$. When using the density functional theory (DFT), it is difficult to model the transition metal oxides [38]. Since the local density (LDA) [39] and the generalized gradient approximations (GGAs) [40] do not consider the exchange and the correlation effects in transition metal oxides thus this leads to important self-interaction errors. For that reason, it is necessary to introduce the selfinteraction correction (SIC) [41], and also the DFT+U [42] methods. On the other hand, the GW approximations (GWA) [43] give more precious results for the compound $\mathrm{CuO}$ [44-45]. The most used compound as a Mott or charge transfer insulator is the compound $\mathrm{CuO}$ in the energy band gap between $1.4-1.7 \mathrm{eV}$ [46-49].

As known in the literature, the $\mathrm{CuO}$ shows a local magnetic moment of $0.65-0.69 \mu \mathrm{B}$ [50], per formula unit. From figure 2(a), it is shown that there is no band gap in the GGA PBE approximation. But, when introducing the SIC approximation, this band gap arises as it is illustrated in figure 2(b). This is due to delocalization of electrons in such materials and nonzero self-interaction errors of DFT. When doping a semiconductor the Fermi level is displaced towards a high energy values. This is due to the fact that the doping action enhances the stability of the compound corresponding to the low energies. Furthermore we used the difference of energy: E$\mathrm{E}_{\mathrm{f}}$. Our results are in good agreement with this statement, see figure 2.

On the other hand, the d-states cross the Fermi level with nonmagnetic states on the $\mathrm{Cu}$ atoms are illustrated in Figure 3. In addition, a direct band gap is shown in this figure at the $\Gamma$ band reached at $1.5 \mathrm{eV}$. The magnetic moment is $0.67 \mu \mathrm{B}$ as it is illustrated in figure 2(b). When exploring the experimental studies, the magnetic 
moment and band gap for $\mathrm{CuO}$ seem to be in good agreement with those found in the literature [46-50]. The transition from ' $d$ ' level to ' $t$ ' and ' $e$ ' parts are separated by the crystal field effects. We illustrate in figure 4 the total and partial density of states (DOS) of the samples for the Fe-doping concentrations ( $5 \%$ and $10 \%$ ). The results are obtained by the GGA PBE and the GGA PBE-SIC approximations (see Figure 4). Indeed, the effect of the crystal field environment is to split the atomic $3 d$ level into five-time degenerate $t$ and $e$ subgroups. The states $t_{2 g}$ and $e_{g}$ are less localized in the valence band states. A near half-metallic behavior is found for the studied of Fe-doped $\mathrm{CuO}$, according to the electronic structure calculations.

In order to highlight the inaccuracy of the GGA PBE, to describe the electronic structure, we introduce the SIC approximation with GGA PBE-SIC. The figure 4 illustrates the near half-metallic behavior in Fe, but no halfmetallic behavior is shown in the compound $\mathrm{Cu}_{1-\mathrm{x}} \mathrm{Fe}_{\mathrm{x}} \mathrm{O}$. This is in contradiction with the other investigation [51]. The $3 d$ levels of Fe-doped $\mathrm{CuO}$ are slightly shifted to a lower energy. This is due to the correction introduced by the SIC-GGA PBE calculations, see figure 4 for $5 \%$ and $10 \%$. The valence band enhances the $p-d$ hybridization according to the GGA-SIC approximation and localizes the $3 d$ impurities level in the bottom of this energy band. Moreover, increasing the Fe-doping effects is to enhance the band gap value from $1.5 \mathrm{eV}$ to $3.1 \mathrm{eV}$.

The Table I resumes the calculated values of the local and total magnetic moments for different concentration values of the Fe-doped $\mathrm{CuO}$ within GGA and GGA-SIC approximations. The total magnetic moment is strongly located in the iron sites, while the local magnetic moment is found at the copper and oxygen atoms. Since the PBE-SIC is a correction of the used model, hence the obtained results are expected to be different than those obtained by the PBE approximation. Therefore, the difference found for the total moment without and within the SIC correction for the concentration $5 \%$ in table 1 , is explained by the magnetic disorder related to the concept of a DLM state. However, two components of one magnetic atom are considered in opposition to the magnetization directions giving rise to the antiferromagnetic state. Otherwise, the other doping ratios 10 and 15 $\%$ correspond to the ferromagnetic case.

TABLE I. Total and local magnetic moment of $\mathrm{Cu}_{1-\mathrm{x}} \mathrm{fe}_{\mathrm{x}} \mathrm{O}$ thin films with GGA and GGA-SIC approximations

\begin{tabular}{|c|c|c|c|c|c|c|c|c|}
\hline \multirow{3}{*}{ Samples } & \multicolumn{4}{|c|}{ PBE } & \multicolumn{4}{|c|}{ PBE-SIC } \\
\hline & \multicolumn{3}{|c|}{ Local moment with GGA } & \multirow[t]{2}{*}{ Total moment } & \multicolumn{3}{|c|}{ Local moment with GGA } & \multirow[t]{2}{*}{ Total momen } \\
\hline & $\mathrm{Cu}$ & $\mathrm{Fe}$ & $\mathrm{O}$ & & $\mathrm{Cu}$ & $\mathrm{Fe}$ & $\mathrm{O}$ & \\
\hline $\mathrm{Cu}_{0.95} \mathrm{Fe}_{0.05} \mathrm{O}$ & 0.00448 & -3.15543 & -0.00835 & -0.3602 & -0.71773 & -3.81780 & -0.32432 & -2.2765 \\
\hline $\mathrm{Cu}_{0.90} \mathrm{Fe}_{0.10} \mathrm{O}$ & -0.00943 & 3.14476 & 0.01773 & 0.7195 & 0.70511 & -3.80297 & 0.22029 & 0.7601 \\
\hline $\mathrm{Cu}_{0.85} \mathrm{Fe}_{0.15} \mathrm{O}$ & -0.01153 & 3.13444 & 0.02771 & 1.0829 & -0.01062 & 3.1467 & 0.0286 & 1.1830 \\
\hline
\end{tabular}

The magnetic energy difference $\Delta \mathrm{E}$ between the FM and the DLM is calculated by:

$$
\Delta \mathrm{E}=\mathrm{E}_{\mathrm{DLM}}-\mathrm{E}_{\mathrm{FM}}
$$

This magnetic disorder is conveniently treated by the concept of a DLM state [52-53]. Two components of one magnetic atom are considered in the DLM states, in opposition to the magnetization directions.

As it is seen in table II, the calculated magnetic energy is compared with GGA and GGA-SIC approximations. From this table, it is found that the introduction of GGA-SIC approximation leads to important values of the energy differences compared to GGA without SIC approximations. Therefore, the critical temperature values are remarkably increased according to equation (2):

$$
\mathrm{T}_{\mathrm{c}}=\frac{2}{3 \mathrm{~K}_{\mathrm{B}}} \frac{\Delta \mathrm{E}}{\mathrm{C}}
$$


Where $\mathrm{C}$ is the doping concentration and $\mathrm{K}_{\mathrm{B}}$ is the Boltzmann constant. The DLM state is described by $\mathrm{Cu}_{1-\mathrm{x}} \mathrm{Fe}_{\mathrm{x} / 2 \uparrow} \mathrm{Fe}_{\mathrm{x} / 2 \downarrow} \mathrm{O}$ and the ferromagnetic state is described by $\mathrm{Cu}_{1-\mathrm{x}} \mathrm{Fe}_{\mathrm{x} \uparrow} \mathrm{O}$.

The paramagnetic states of ferro-magnets are well described by the DLM states, see Ref. [54].

From the table II, it is well seen that the ferromagnetic state is the stable one for the Fe-doped CuO compound. Regarding the Katayama- Yoshida and Sato rule, the $\Delta \mathrm{E}$ values are better described in the density of states.

The system exhibit a ferromagnetic state since its energy is so that: $\Delta \mathrm{E}>0$ which corresponds to the ferromagnetic phase, see table II.

Within GGA-SIC Approximation, it is well known that the more positive larger $\Delta \mathrm{E}$ corresponds to the more stable ferromagnetic state of the studied $\mathrm{Fe}$ doped $\mathrm{CuO}$ components.

The Curie temperature $\left(T_{C}\right)$ is investigated from the total energy difference $\Delta E$ according to equation (2), in the framework of the Mean Field Approximation (MFA).

Table II summarizes the calculated values of $T_{c}$ by using MFA approximation. The square root of the concentration tendency is revealed. From this table, the increasing concentration values of iron leads to increasing values of $\mathrm{T}_{\mathrm{c}}$, in the PBE-SIC approximation. On the other hand, the PBE approximation without SIC correction reveals important values of $\mathrm{T}_{\mathrm{c}}$ for very low concentration values, as it is illustrated in table II.

TABLE II. The relative energies of FM and DLM orderings of $\mathrm{Cu}_{1-x} \mathrm{Fe}_{\mathrm{x}} \mathrm{O}$ thin films with GGA and GGA-SIC approximations.

\begin{tabular}{|l|c|c|c|c|}
\hline \multirow{2}{*}{ Samples } & \multicolumn{2}{|c|}{ PBE } & \multicolumn{2}{c|}{ PBE-SIC } \\
\cline { 2 - 5 } & $\Delta \mathbf{E}\left(\mathbf{E}_{\text {DLM- }}-\mathbf{E}_{\text {Ferr }}\right)(\mathrm{eV})$ & $\mathbf{T}_{\mathbf{C}}\left({ }^{\circ} \mathrm{K}\right)$ & $\Delta \mathbf{E}\left(\mathbf{E}_{\mathbf{D L M}}-\mathbf{E} \mathbf{E}_{\text {Ferr }}\right)(\mathrm{eV})$ & $\mathbf{T}_{\mathbf{C}}\left({ }^{\circ} \mathrm{K}\right)$ \\
\hline $\mathrm{Cu}_{0.95} \mathrm{Fe}_{0.05} \mathrm{O}$ & 0.002321132 & 340.168 & 0.002585083 & 255.614 \\
\hline $\mathrm{Cu}_{0.90} \mathrm{Fe}_{0.10} \mathrm{O}$ & 0.003153801 & 487.939 & 0.003254483 & 503.516 \\
\hline $\mathrm{Cu}_{0.85} \mathrm{Fe}_{0.15} \mathrm{O}$ & 0.003289858 & 492.939 & 0.003361968 & 553.406 \\
\hline
\end{tabular}

\subsection{Absorption Spectra}

Optical spectroscopy is another powerful method that allows the study of the electronic structure of crystals; this method has its advantages to complete the physical interpretation of the extracted information from the density-of-state (DOS). The absorption spectra of $\mathrm{Cu}_{1-\mathrm{x}} \mathrm{Fe}_{\mathrm{x}} \mathrm{O}$ within GGA PBE and GGA PBE -SIC approximations are shown in Figure 5. It corresponds to transitions of 1s electrons of TM to empty states above the Fermi level. Meanwhile, other transitions are observed in this energy range as the transitions of $2 \mathrm{~s}$ electrons. These transitions give only a very smooth contribution to the absorption spectrum while the absorption caused by transitions of $1 \mathrm{~s}$ electrons changes very much near the absorption K-edge. That explains why the structure of the K-edge absorption spectra is determined by transitions of 1 s electrons. Using the dipole approximation and taking into account these transitions, one can evaluate the absorption coefficients $\mu(\mathrm{E})$ [55-56]:

$$
\mu(\mathrm{E}) \infty\left|\left\langle\Psi_{1 \mathrm{~s}}|\mathrm{e} \nabla| \Psi_{4 \mathrm{p}}\right\rangle\right|^{2} \cdot \mathrm{n}_{4 \mathrm{p}}\left(\hbar \omega-\mathrm{E}_{\mathrm{F}}+\mathrm{E}_{1 \mathrm{~s}}\right)
$$

Where: $\Psi_{1 \mathrm{~s}}$ is the wave function of a 1 s electron, $\Psi_{4 \mathrm{p}}$ is the wave function of a $4 p$ empty state, $\mathrm{n}_{4 \mathrm{p}}$ is the density of $4 p$ states above the Fermi level, e is the light polarization.

When Fe atom is in a substitution position in the host semiconductor $\mathrm{CuO}$, it is surrounded by six oxygen atoms located on the vertices of an octahedron environment. The octahedral crystal field of oxygen ions splits $3 \mathrm{~d}$-states of $\mathrm{Fe}$, which are mainly located in the band gap, into $e_{g}$ and $t_{2 g}$ levels with $e_{g}$ below $t_{2 g}$ [57-59]. Exchange interactions further split these states into spin-up $(\uparrow)$ and spin-down $(\downarrow)$ states. Due to the tetrahedral arrangement of the ligands, the $\mathrm{t}_{2 \mathrm{~g}}$-states of the Fe atoms hybridize with $\mathrm{Fe} 4 \mathrm{p}$-states. The states resulting from hybridization 
of the deeper $t_{2 g} \uparrow$ - states show mainly Fe $-3 d$ character. From Figure 5, the outline of the main result and comparison is as follows: first the Fe-doped $\mathrm{CuO}$ K-edge within GGA PBE and GGA PBE -SIC exhibit a weak shoulder on a rising absorption curve that culminates in a strong peak. This shoulder is caused by the transition $1 \mathrm{~s}$ to $4 \mathrm{p}$ that is authorized as an effect of the $4 \mathrm{p}-3 \mathrm{~d}$ mixing due to the $4 \mathrm{p}-3 \mathrm{~d}$ hybridization. In fact, the discrepancy between the results obtained between GGA and GGA-SIC results: in the first case, the correlation effects are not treated properly. In the second case, the localization of the $4 p$ orbital of $\mathrm{Fe}$ in the valence band is overestimated. The shape of the spectra does not depend on the Fe content in $\mathrm{CuO}$. Therefore, the Fe atoms have the same valence and local crystal structure in all the samples.

(a)

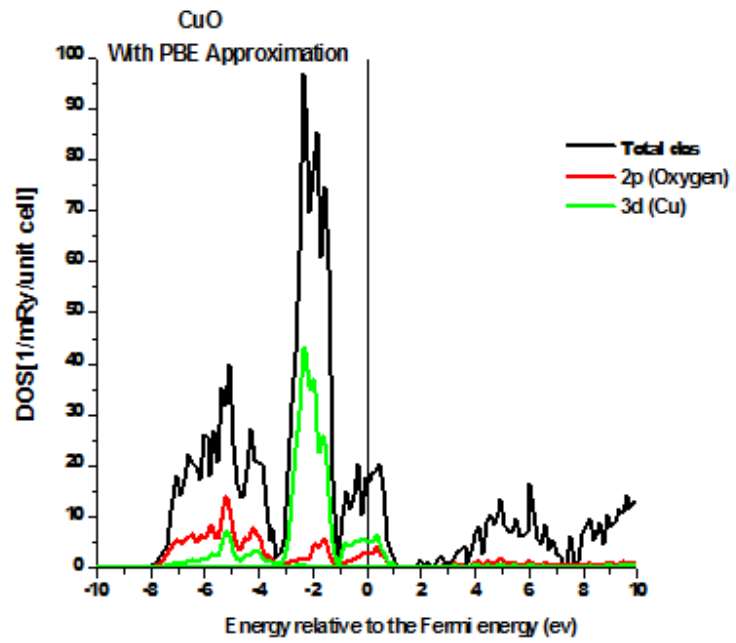

(b)

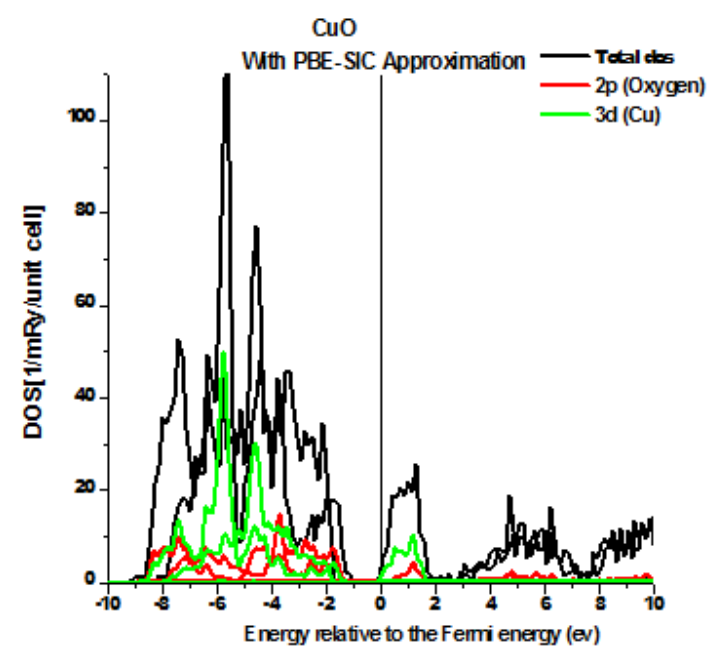

Figure 2. The total projected DOS of $\mathrm{CuO}$ within GGA and GGA-SIC

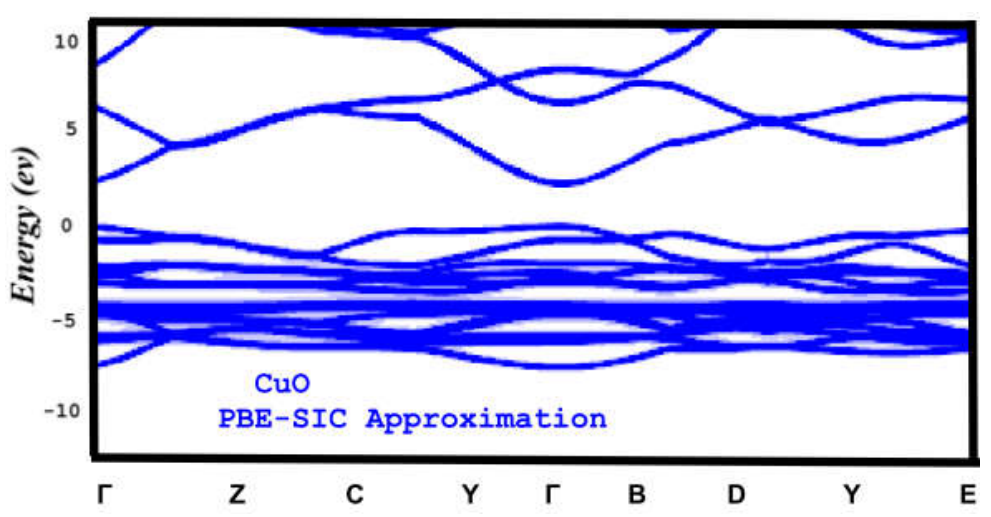

Figure 3. The band structure of $\mathrm{CuO}$ within GGA-SIC 

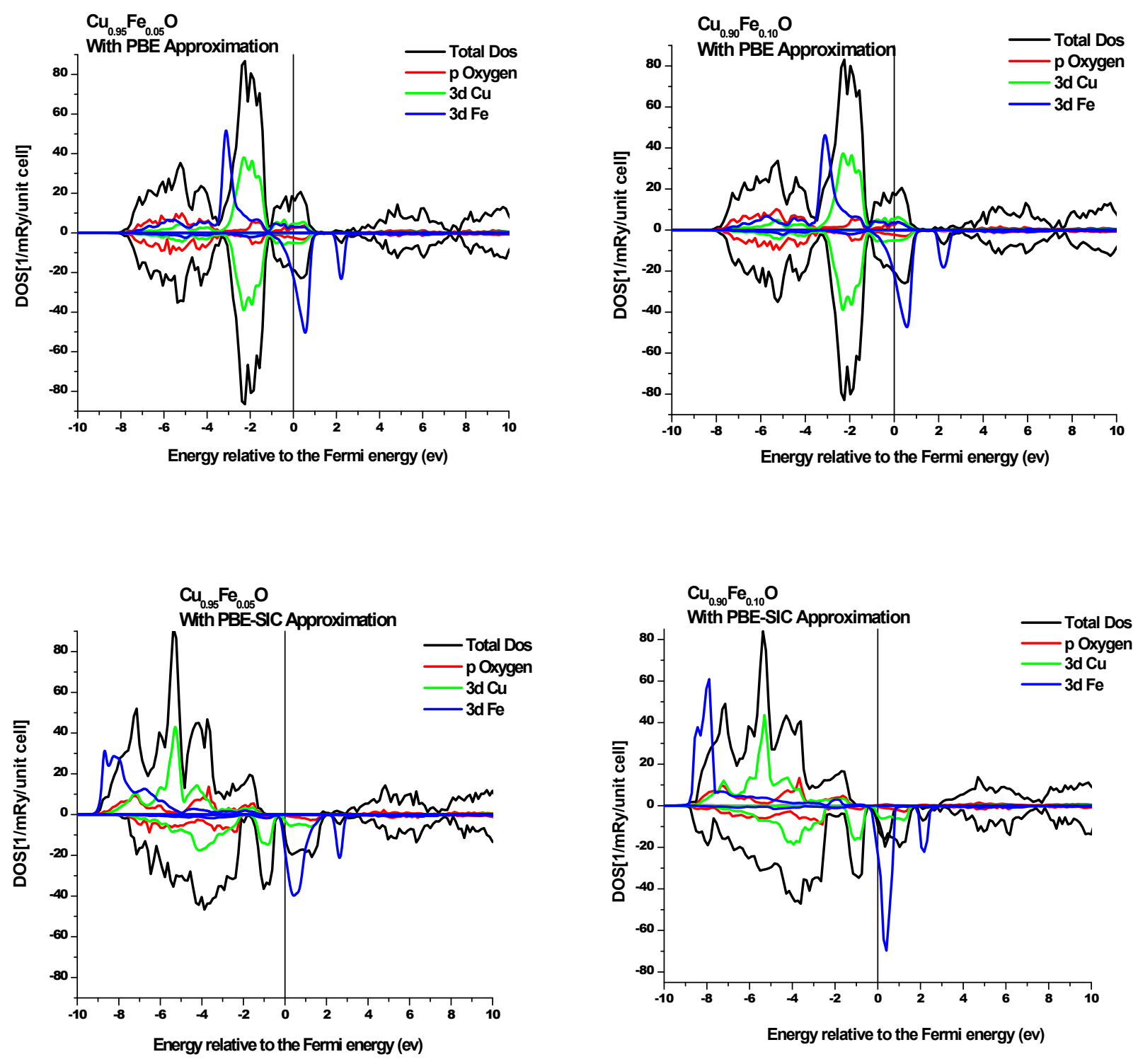

Figure 4. The total and $\mathrm{d}(\mathrm{TM})$ states projected DOS of $\mathrm{CuO}$ within GGA and GGA-SIC approximations 

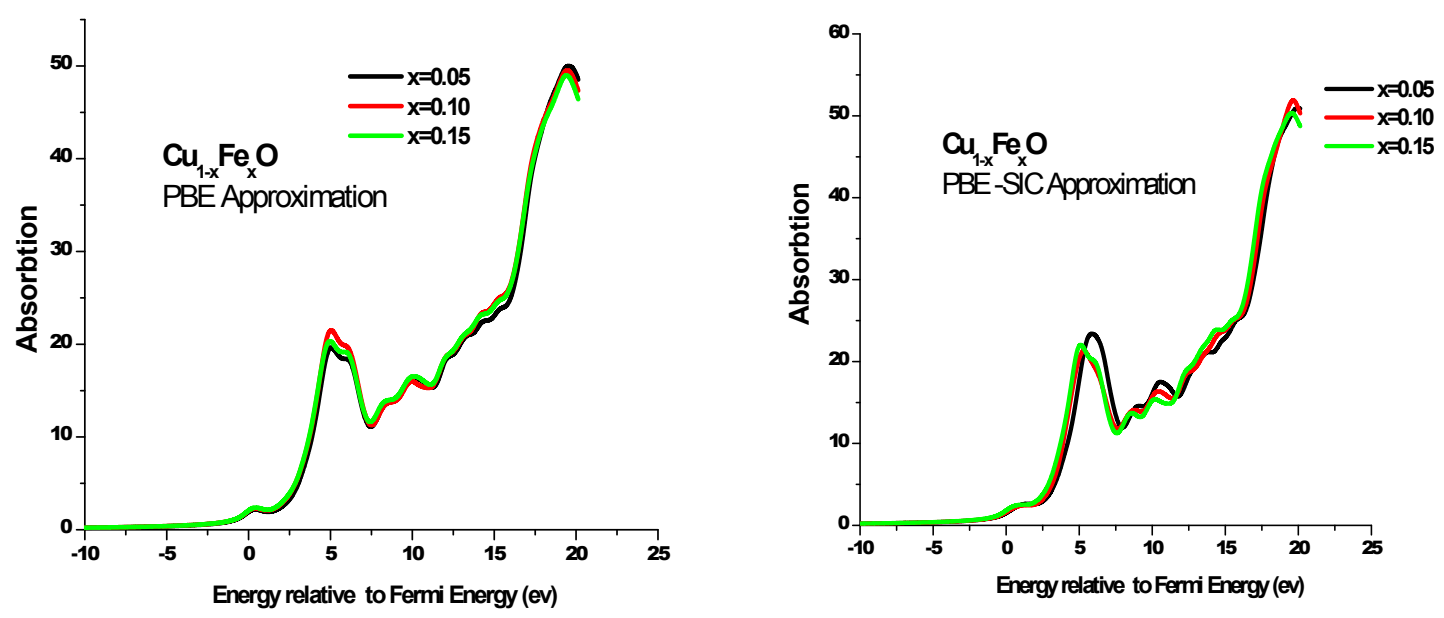

Figure 5. The calculated absorption spectrum at the K-edge $\mathrm{CuO}$ with GGA and GGA-SIC approximations

\section{Conclusion}

The electronic structures for $\mathrm{Cu}_{1-\mathrm{x}} \mathrm{Fe}_{\mathrm{x}} \mathrm{O}$ have been investigated using the KKR-CPA method in connection with GGA without and within SIC corrections, respectively. The study of the electronic structure, the magnetic moment and absorption spectra of the investigated films with different concentration of iron Fe have been performed and discussed. Compared with the pure $\mathrm{CuO}$, the Fermilevels of the doped structures move to the higher energy directions. Finally, the increasing concentration of $\mathrm{Fe}$-doping $\mathrm{CuO}$ can render the material half-metallic with a high wide impurity band in the framework of the two approximations LDA and SIC.

\section{REFERENCES}

[1]. V.K. Sharma, R. Xalxo, G.D. Varma, Structural and magnetic studies of Mn-doped ZnO, Cryst. Q6 Res. Technol. 42 (2007) 34

[2]. Kimura, T, Y. Sekio, H. Nakamura, T. Siegrist, and A. P. Ramirez. Cupric oxide as an induced-multiferroic with high-TC. Nature materials 7, no. 4 (2008) 291-294.

[3]. M.A. Dar, Y.S. Kim, W.B. Kim, J.M. Sohn, H.S. Shin, Appl. Surf. Sci. 254 (2008) 7477.

[4]. Chafi, F.Z., Jabar, A., Bahmad, L. et al. J Supercond Nov Magn (2017). doi:10.1007/s10948-017-3995-7

[5]. R. N. Mariammal, K. Ramachandran, G. Kalaiselvan, S. Arumugam, B. Renganathan, D. Sastikumar, Effect of magnetism on the ethanol sensitivity of undoped and Mn-doped CuO nanoflakes, Appl. Surf. Sci. $270(2013) 545-552$.

[6]. S. Manna, S. K. De, Room temperature ferromagnetism in Fe doped CuO nanorods, J. Magnet. Magnet. Mater. 322(2010)2749-2753. 
[7]. K. L. Liu, S. L. Yuan, H. N. Duan, X. F. Zheng, S. Y. Yin, Z. M. Tian, C. H. Wang, S. X. Huo, Exchange bias in Fe and Ni codoped CuO nanocomposites, J. Appl. Phys. 107(2010)023911.

[8]. Tu, C.H, et al, C. P. (2014). Resistive memory devices with high switching endurance through single filaments in Bi-crystal $\mathrm{CuO}$ nanowires. Journal of Alloys and Compounds, 615, 754-760.

[9]. Luo, Lin-Bao, Xian-He Wang, Chao Xie, Zhong-Jun Li, Rui Lu, Xiao-Bao Yang, and Jian Lu. "Onedimensional $\mathrm{CuO}$ nanowire: synthesis, electrical, and optoelectronic devices application." Nanoscale research letters 9 , no. 1 (2014): 1-8.

[10]. Li, Dongdong, Jun Hu, Ruqian Wu, and Jia G. Lu. "Conductometric chemical sensor based on individual CuO nanowires." Nanotechnology 21, no. 48 (2010): 485502.

[11]. G. Zhu, H. Xu, Y. Xiao, Y. Liu, A. Yuan, X. Shen, Facile fabrication and enhanced sensing properties of hierarchically porous $\mathrm{CuO}$ architectures, ACS Appl. Mater. Interfaces 4(2012)744-751.

[12]. K. Jindal, M. Tomar, V. Gupta, CuO thin film base duric acid bio sensor with enhanced response characteristics, Biosens. Bio electron. 38(2012)11-18.

[13]. D. Jundale, S. Pawar, M. Chougule, P. Godse, S. Patil, B. Raut, S. Sen, V. Patil, Nanocrystalline CuO thin films for H2S monitoring: micro structuraland optoelectronic characterization, J. Sensor Technol. 1(2011)3646.

[14]. M. H. Habibi, B. Karimi, M. Zendehdel, M. Habibi, Fabrication, characterization of two nano-composite $\mathrm{CuO}-\mathrm{ZnO}$ working electrodes for dye-sensitized solar cell, Spectrochim. Acta A116 (2013)374-380.

[15]. R. Sivaraj, P. K. S. M. Rahman, P. Rajiv, H. Abdul Salam, R. Venckatesh, Biogenic copper oxide nanoparticles synthesis using Tabernaemontanadivaricate leaf extract and its antibacterial activity agains turinarytract pathogen, Spectrochim. Acta A133 (2014)178-181.

[16]. J. S. Shaikh, R. C. Pawar, R. S. Devan, Y. R. Ma, P. P. Salvi, S. S. Kolekar, P. S. Patil, Synthesisand characterization of $\mathrm{Ru}$ doped $\mathrm{CuO}$ thin films for super capacitor Based on Bronsted acidic ionic liquid, Electrochim. Acta 56(2011)2127-2134.

[17]. M. Umadevi, A. J. Christy, Synthesis, characterization and photocatalytic activity of CuO nanoflowers, Spectrochim. ActaA109(2013)133-137.

[18]. R. Sankar, P. Manikandan, V. Malarvizhi, T. Fathima, K. S. Shivashangari, V. Ravikumar, Green synthesis of colloidal copper oxide nanoparticles using Caricapapaya and its application in photocatalytic dye degradation, Spectrochim. Acta A121(2014)746-750.

[19]. N. Mukherjee, B. Show, S. K. Maji, U. Madhu, S. K. Bhar, B. C. Mitra, G. G. Khan, A. Mondal, CuO nanowhiskers: electrodeposition, Ramananalysis, photoluminescence study and photocatalytic activity, Mater. Lett. 65(2011)3248-3250.

[20]. Q. Zhang, K. Zhang, D. Xu, G. Yang, H. Huang, F. Nie, C. Liu, S. Yang, CuO nanostructures: synthesis, characterization, growth mechanisms, fundamental properties and applications, Progr. Mater. Sci. 60(2014)208-337. 
[21]. M. -Hui Chang, H. -Shen Liu, C. Y. Tai, Preparation of copper oxide nanoparticles and its application in nanofluid, Powder Technol. 207(2011)378-386.

[22]. R. Sivaraj, P. K. S. M. Rahman, P. Rajiv, S. Narendhran, R. Venckatesh, Bio synthesis and characterization of Acalypha in dicamediated copperoxide nanoparticles and evaluation of its antimicrobial and anticancer activity, Spectrochim. Acta A129(2014)255-258.

[23]. A. P. Moura, L. S. Cavalcante, J. C. Sczancoski, D. G. Stroppa, E. C. Paris, A. J. Ramirez, J. A. Varela, E. Longo, Structure and growth mechanism of $\mathrm{CuO}$ plates obtained by microwave-hydrothermal without surfactants, Adv. Powder Technol. 21(2010)197-202.

[24]. G.V. Cherithin, L. Andrews, C.W. Bauschlicher Jr, J. Phys. Chem. A 101 (1997) 4026.

[25]. Y. Pouillion, C. Massobrio, M. Celino, Comput. Mater. Sci. 17 (2000) 539.

[26]. A. Daoudi, A.T. Benjelloun, J.P. Flament, G. Berthier, J. Mol. Spectrosc.194 (1999) 8.

[27]. Y. Mochizuki, K. Tanaka, H.K. Kashiwagi, Chem. Phys. 151 (1991) 11.

[28]. L.S. Wang, H. Wu, S.R. Desai, L. Lou, Phys. Rev. B 53 (1996) 8028.

[29]. T. Baruah, R.R. Zope, M.R. Pederson, Phys. Rev. A 69 (2004) 023201.

[30]. J. P. Perdew, J. A. Chevary, S. H. Vosko, K. A. Jackson, M. R. Pederson, D. J. Singh, and C. Fiolhais; Phys. Rev. B, 46, 6671 (1992).

[31]. H. Akai: http://sham.phys.sci.osaka-u.ac.jp/ kkr/.

[32]. M. Toyoda, H. Akai, K. Sato, and H. Katayama-Yoshida: Physica B376 (2006) 647.

[33]. J.P. Perdew, A. Zunger, Phys. Rev. B 23 (1981) 5048.

[34]. Sato, K., Dederichs, P.H., Katayama-Yoshida, H.: Physica B 376/377, 639 (2006)

[35]. H. Akai: Phys. Rev. Lett. 81 (1998) 3002

[36]. H. Akai, "MACHIKANEYAMA2002v08, Department of Physics, Graduate School of Science, Osaka University, Japan (akai@phys.sci.osaka-u.ac.jp).”

[37]. Samir Paudel, Sushmita Dandeliya, Rajneesh Chaurasiya, Anurag Srivastava and Gopi Kaphle, Magnetism in Zigzag and Armchair CuO Nanotubes: Ab-Initio Study, Journal of Magnetism and Magnetic Materials, j.jmmm.2015.12.081

[38]. Kohn, W.; Sham, L. D.Self-Consistent Equations Including Exchange and Correlation Effects Phys. Rev. 1965, 140, A1133- A1138, DOI: 10.1103/PhysRev.140.A1133

[39]. Perdew, J. P.; Burke, K.; Ernzerhof, M.Generalized Gradient Approximation Made Simple Phys. Rev. Lett. 1996, 77, 3865- 3868, DOI: 10.1103/PhysRevLett.77.3865

[40]. Perdew, J. P.; Zunger, A.Self-interaction Correction to Density-Functional Approximations for ManyElectron Systems Phys. Rev. B: Condens. Matter Mater. Phys. 1981, 23, 5048- 5079, DOI: 10.1103/PhysRevB.23.5048 
[41]. Hedin, L.New Method for Calculating the One-Particle Green's Function with Application to the ElectronGas Problem Phys. Rev. 1965, 139, A796- A823, DOI: 10.1103/PhysRev.139.A796

[42]. Svane, A.; Gunnarsson, O.Transition-Metal Oxides in the Self-Interaction-Corrected Density-Functional Formalism Phys. Rev. Lett. 1990, 65, 1148- 1151, DOI: 10.1103/PhysRevLett.65.1148

[43]. Takahashi, M.; Igarashi, J. I.Electronic Excitations in Cupric Oxide Phys. Rev. B: Condens. Matter Mater. Phys. 1997, 56, 12818-12824, DOI: 10.1103/PhysRevB.56.12818

[44]. Dudarev, S. L.; Botton, G. A.; Savrasov, S. Y.; Humphreys, C. J.; Sutton, A. P.Electron-Energy-Loss Spectra and the Structural Stability of Nickel Oxide: An LSDA+U Study Phys. Rev. B: Condens. Matter Mater. Phys. 1998, 57, 1505- 1509, DOI: 10.1103/PhysRevB.57.1505

[45]. Ghijsen, J.; Tjeng, L.; Elp, J. V.; Eskes, H.; Westerink, J.; Sawatzky, G.; Czyzyk, M.Electronic Structure of $\mathrm{Cu} 2 \mathrm{O}$ and $\mathrm{CuO}$ Phys. Rev. B: Condens. Matter Mater. Phys. 1988, 38, 11322- 11330, DOI: 10.1103/PhysRevB.38.11322

[46]. Chaudhary, Y. S.; Agrawal, A.; Shrivastav, R.; Satsangi, V. R.; Dass, S.A Study on the Photoelectrochemical Properties of Copper Oxide Thin Films Int. J. Hydrogen Energy 2004, 29, 131- 134, DOI: $10.1016 / \mathrm{S} 0360-3199(03) 00109-5$

[47]. Koffyberg, F. P.; Benko, F. A.A Photoelectrochemical Determination of the Position of the Conduction and Valence Band Edges of p-type CuO J. Appl. Phys. 1982, 53, 1173-1177, DOI: 10.1063/1.330567

[48]. Marabelli, F.; Parravicini, G.; Salghetti, D. F.Optical Gap of CuO Phys. Rev. B: Condens. Matter Mater. Phys. 1995, 52, 1433- 1436, DOI: 10.1103/PhysRevB.52.1433

[49]. Forsyth, J. B.; Brown, P. J.; Wanklyn, B. M.Magnetism in Cupric Oxide J. Phys. C: Solid State Phys. 1988, 21, 2917- 2929, DOI: 10.1088/0022-3719/21/15/023

[50]. Yang, B. X.; Thurston, T. R.; Tranquada, J. M.; Shirane, G.Magnetic Neutron Scattering Study of SingleCrystal Cupric Oxide Phys. Rev. B: Condens. Matter Mater. Phys. 1989, 39, 4343- 4349, DOI: 10.1103/PhysRevB.39.4343

[51]. T. Oguchi, K. Terakura, and N. Hamada,J. Phys. F13, 145 (1983)

[52]. B. L. Gyorffy, A. J. Pindor, J. Staunton, G. M. Stocks, and H. Winter,J. Phys. F15, 1337 (1985)

[53]. Metropolis, N., \& Ulam, S. (1949). The monte carlo method. Journal of the American statistical association, 44(247), 335-341

[54]. B. Beschoten, P. A. Crowell, I. Malajovich, D. D. Awschalom, F. Matsukura, A. Shen and H. Ohno, Magnetic circular dichroism studies of carrier-induced ferromagnetism in (Ga1-xMnx)As, Phys. Rev. Lett. 83, 3073 (1999)

[55]. L. Bergqvist, O. Eriksson, J. Kudrnovský, V. Drchal, P. A. Korzhavyi and I. Turek, Phys. Rev. Lett. 93, 137202 (2004).

[56]. K. Sato, W. Schweika, P. H. Dederichs and H. Katayama-Yoshida, Phys. Rev. B 70, 201202(R)(2004)

[57]. P. Mahadevan, A. Zunger, Phys. Rev. B 68 (2003) 075202 
[58]. P. Mahadevan, A. Zunger, Phys. Rev. B 69 (2004) 115211

[59]. Binder, K. Introduction: Theory and "technical" aspects of Monte Carlo simulations Springer Berlin Heidelberg. (1986) (pp. 1-45). 